\title{
Médicaments Off-label use
}

Beat Seiler ${ }^{a}$, Robert Fries ${ }^{b}$, Hanspeter Honegger ${ }^{c}$

a Dr, médecin-conseil en chef de Helsana Groupe

b Dr, médecin-conseil de Helsana Groupe

c Prof. Dr, médecin spécialiste en oncologie/hématologie, FMH
En 2011, les médecins-conseils de Helsana ont développé MediScore, un modèle d'évaluation du bénéfice des médicaments. Depuis 2012, toutes les demandes selon l'art. 71 a/b OAMal sont désormais systématiquement évaluées à l'aide de MediScore. L'analyse des statistiques 2012 de Helsana montre que les résultats sont applicables en pratique et que les assurés ont ainsi rapidement accès à des traitements innovants et acceptables d'un point de vue éthique. L'affectation à une catégorie de bénéfices permet de déduire un modèle de remboursement échelonné. Les médicaments anticancéreux ne représentent que 30\% des demandes selon l'art. 71, mais génèrent la quote-part de frais la plus importante. L'autorisation administrative doit être améliorée pour que les indications fréquentes puissent être enregistrées rapidement dans la LS. MediScore fait aujourd'hui partie intégrante d'une solution telle que la conseille la Société Suisse des médecins-conseils et médecins d'assurances (SSMC).

\section{MediScore repose sur \\ l'evidence-based medicine.}

\section{Contexte}

Fin 2011, les médecins-conseils de Helsana ont commencé à examiner systématiquement les demandes de traitements médicamenteux en dehors de l'autorisation régulière avec la grille de MediScore [1]. Le médecin-conseil est au cœur de l'art. 71 a/b OAMal. C'est à lui qu'il appartient de décider s'il faut s'attendre à un bénéfice thérapeutique important au cas par cas. Pour cette évaluation, il a besoin de données relatives à l'efficacité d'un médicament qu'il trouve dans des études et d'informations sur la situation clinique fournies par le médecin traitant. Le modèle de MediScore autorise une évaluation simple et rapide de l'effet d'un médicament dans le collectif étudié. Le médecin-conseil peut déterminer le bénéfice thérapeutique escompté selon l'adéquation du cas individuel avec le collectif étudié.

Le modèle MediScore a été présenté publiquement à plusieurs reprises [2] et il est aujourd'hui consultable sur le site Internet de la SSMC [1]. La grande inconnue est de savoir si un tel modèle de bénéfice fonctionne en pratique. Helsana est le premier assureur important à pouvoir présenter une statistique relative à l'art. 71 datant de 2012, qui se fonde sur l'évaluation systématique du bénéfice avec MediScore.

\section{MediScore}

La gestion de la problématique des médicaments orphelins et hors étiquette en termes de bénéfices et de tarification n'a pas encore été résolue de manière satisfaisante en Suisse, mais aussi dans le monde [4]. MediScore permet certes de catégoriser l'importance du bénéfice et d'en déduire une rétribution financière, mais il n'y a pas en Suisse d'obligation de travailler avec un modèle. Il en résulte des inégalités de traitement, comme celle dénoncée par la Ligue contre le cancer. MediScore qui fait partie de la solution SSMC depuis janvier 2013 [1] est un instrument fiable permettant de gérer ce problème pour que les patients concernés bénéficient de traitements innovants et acceptables au plan éthique dans un système de santé social. La catégorisation selon l'importance du bénéfice permet à l'assureur de déduire un remboursement financier approprié, comme l'exige l'art. $71 \mathrm{a} / \mathrm{b}$ OAMal.

L'art. 71 a/b OAMal prévoit une bipartition. Le médecin-conseil de l'assurance-maladie détermine l'importance du bénéfice et l'assurance en déduit le remboursement. L'acceptation du modèle par le corps médical et l'industrie pharmaceutique est nécessaire pour la gestion au quotidien. Ce n'est possible que parce que MediScore est transparent et reproductible. Tous les paramètres d'étude importants ont par ailleurs été intégrés dans le modèle. Les centaines d'applications attestent le réalisme des résultats. L'évaluation se fonde sur les principes de l'evidencebased medicine, parce que la meilleure preuve scientifique actuelle est utilisée en combinaison avec l'expérience pratique.

\section{Classification avec MediScore}

Le Score-Rating d'une étude se réfère à l'efficacité du médicament dans le collectif étudié. Le médecinconseil doit déterminer si la situation individuelle du patient est conforme à la situation dans le collectif étudié. Les informations fournies par le médecin traitant servent de base. L'expérience montre que dans plus de $90 \%$ des demandes, le cas individuel correspond au collectif étudié et que la classification des études représente donc aussi la classification du bénéfice. Si la situation individuelle du patient diffère fortement de la situation décrite du collectif étudié, le médecin-conseil doit décider si une correction 


\begin{tabular}{llll}
$\begin{array}{l}\text { Tableau } 1 \\
\text { Classification. }\end{array}$ & & & \\
Catégorie & Points de score & Paiement par l'assurance-maladie & Remarques \\
\hline A & $20-27$ & Oui & \\
\hline B & $13-19$ & Oui & $\begin{array}{l}\text { Oui, si essai thérapeutique } \\
\text { réussi }\end{array}$ \\
C & $6-12$ & Non & \\
\hline D & $0-5$ & Non &
\end{tabular}

de la classification du bénéfice est indiquée. Dans le doute, il optera pour la catégorie C.

Dans les situations orphelines, telles que les maladies orphelines ou les indications orphelines, il n'y a pas toujours d'essai clinique randomisé (ECR) et les points de score obtenus sont souvent insuffisants pour indiquer une grande efficacité. Si seul un résultat d'étude inférieur est attesté, mais qu'il y a de forts indices d'un important bénéfice éventuel dans un cas particulier, nous recommandons un essai thérapeutique pour des raisons éthiques (catégorie C). L'assurance-maladie ne peut certes pas prendre en charge ces coûts, mais des accords avec les sociétés contacté. Dans les cas d'étude difficiles, le classement a été vérifié par un deuxième médecin-conseil. Lors de l'évaluation des essais thérapeutiques de la catégorie $\mathrm{C}$, nous nous sommes appuyés sur les indications des médecins traitants.

Le tableau 2 montre les résultats de classification du bénéfice. Il montre également qu'un bénéfice important a pu être attesté lors de l'évaluation initiale dans $60 \%$ des demandes (A et $B$ ). Un grand bénéfice a ultérieurement été démontré dans 121 cas dans le cadre d'un essai thérapeutique sur la base de la catégorie $\mathrm{C}$, de sorte qu'un grand bénéfice a globalement été confirmé dans 66\% des 1956 demandes en 2012.

\section{Dans $66 \%$ de toutes les demandes, un important bénéfice} thérapeutique a été attesté.

pharmaceutiques qui fournissent les médicaments pour l'essai thérapeutique sont possibles. Il est rare que les assurés aient à supporter les coûts eux-mêmes. S'il s'avère au cours du traitement que celui-ci fonctionne, l'important bénéfice est démontré dans le cas particulier et l'assurance-maladie prend en charge la suite du traitement.

\section{Statistique 2012 de Helsana}

Sur la base de MediScore, Helsana a procédé à une évaluation des demandes de médicaments selon l'art. 71 a/b OAMal dans 1956 cas en 2012.

Au plan méthodologique, nous avons fait en sorte que toutes les demandes soient évaluées au fur et à mesure de leur réception sans sélection préalable. De nombreux cas ont pu être réglés par le biais d'une liste de classification interne (base de données) pour les médicaments récurrents et leurs indications. Après vérification des conditions de l'art. 71 et rapprochement de la situation concrète du patient avec le collectif étudié, la classification de la base de données a été utilisée et transmise à la gestion des prestations pour la garantie de prise en charge des coûts. Les cas en dehors du classement ont été notés selon les propres évaluations des études avec la meilleure étude. Dans le doute, le médecin traitant a été
Le tableau 3 montre que le grand nombre de demandes (70\%) concerne le domaine hors oncologie. $21 \%$ des demandes concernent l'art. 71 b OAMal. Il n'y a pas de fixation des prix par les autorités dans ce domaine.

Il est flagrant dans le tableau 4 que des cas nettement plus nombreux sont rejetés en dehors de l'oncologie. Cela s'explique par le fait que ce n'est pas tant le risque vital qui importe en dehors de l'oncologie que l'atteinte grave et chronique. Or il manque à ce jour une définition de ce qu'est une atteinte grave et chronique au sens de l'art. 71 OAMal. Les premiers jugements rendus par les tribunaux indiquent qu'elle doit impliquer une restriction des activités de la vie courante (ADL) (5).

Le tableau 5 montre que la plupart des médicaments du TOP 20 ne sont pas des médicaments anticancéreux. Nous pensons que les médicaments qui sont très souvent réglés par le biais de l'art. 71 (rapport inférieur à 1:10) devraient être rapidement admis dans la liste des spécialités.

Le tableau 6 montre que les coûts des médicaments selon l'art. 71 concernent majoritairement les médicaments anticancéreux. Seules six préparations du TOP 20 sont des médicaments exclusivement non anticancéreux. 
Tableau 2

Evaluation du bénéfice des médicaments avec MediScore, Helsana 2012.

\begin{tabular}{|c|c|c|c|c|c|c|}
\hline $\begin{array}{l}\text { Nombre de cas } \\
\text { (n) }(\%)\end{array}$ & A & B & $C^{*}$ & D & Rejets ** & \\
\hline 1956 (100\%) & 740 (38\%) & $438(22 \%)$ & 447 (23\%) & 19 (1\%) & $312(16 \%)$ & \\
\hline $\begin{array}{l}\text { * L'essai thérapeuti } \\
\text { ** Rejet, parce que } \\
\rightarrow \text { il existe une } \\
\rightarrow \text { il n'y a pas d } \\
\rightarrow \text { médicament }\end{array}$ & $\begin{array}{l}\text { ait réussi dans } \\
\text { nditions de l'ar } \\
\text { tive thérapeuti } \\
\text { die grave et chr } \\
\text { ger utilisé hors }\end{array}$ & $\begin{array}{l}\text { cas ( } 27 \%) \text {. } \\
\text { a/b OAMal ne } \\
\text { efficace et auto } \\
\text { que ou potentie } \\
\text { dette. }\end{array}$ & $\begin{array}{l}\text { pas remplies. } \\
\text { ent mortelle. }\end{array}$ & & & (Pourcentages arrondis) \\
\hline
\end{tabular}

Tableau 3

Répartition des cas et des médicaments selon l'art. 71, Helsana 2012.

\begin{tabular}{llll} 
Domaine & Nombre de médicaments & $\begin{array}{l}\text { Nombre de cas } \\
\text { Art. } 71 \text { a et b }\end{array}$ & $\begin{array}{l}\text { Nombre de cas concernant } \\
\text { uniquement l'art. } 71 \text { b }\end{array}$ \\
\hline Oncologie & 96 & $579(30 \%)$ & 87 \\
\hline Hors oncologie & 188 & $1377(70 \%)$ & 331 \\
\hline Total & 284 & $1956(100 \%)$ & $418(21 \%)$ \\
\hline & & & (Pourcentages arrondis)
\end{tabular}

Tableau 4

Evaluation du bénéfice anticancéreux/non anticancéreux, Helsana 2012.

\begin{tabular}{|c|c|c|c|c|c|}
\hline $\begin{array}{l}\text { Nombre de cas } \\
\text { Oncologie }\end{array}$ & A & B & C & D & Rejets \\
\hline $579(100 \%)$ & $210(36 \%)$ & $201(35 \%)$ & $112(19 \%)$ & $7(1 \%)$ & $49(9 \%)$ \\
\hline \multicolumn{6}{|c|}{$\begin{array}{l}\text { Nombre de cas } \\
\text { non anticancéreux }\end{array}$} \\
\hline 1377 (100\%) & $530(39 \%)$ & $23(17 \%)$ & $335(24 \%)$ & 12 (1 \%) & $263(19 \%)$ \\
\hline
\end{tabular}

\section{Tableau 5}

TOP 20 des applications de médicaments selon l'art. 71 et de façon régulière, Helsana 2012.

\begin{tabular}{|lllll} 
Rang & Médicament & $\begin{array}{l}\text { Nombre de cas } \\
\text { selon l'art. 71 }\end{array}$ & $\begin{array}{l}\text { Nombre de cas } \\
\text { dans une application régulière }\end{array}$ & $\begin{array}{l}\text { Rapport d'application: } \\
\text { art }\end{array}$ \\
\hline 1 & Botox & 148 & 543 & $1: 3,6$ \\
\hline 2 & Mabthera** & 133 & 511 & $1: 3,8$ \\
\hline 3 & Avastin** & 113 & 420 & $1: 3,7$ \\
\hline 4 & Ritalin & 55 & 1896 & $1: 34$ \\
\hline 5 & Ribomustin* & 52 & 91 & $1: 1,7$ \\
\hline 6 & Cellcept & 43 & 729 & $1: 17$ \\
\hline 7 & Zytiga* & 34 & 100 & $1: 3$ \\
\hline 8 & Dronabinol & 28 & Impossible à déterminer & - \\
\hline 9 & Revlimid* & 27 & 146 & $1: 5,4$ \\
\hline 10 & Remicade & 26 & 665 & $1: 25$ \\
\hline $10-12$ & Divers & 196 & Jevtana*, Victoza, Afinitor*, Recormon, Prolia, Fampyra, Xolair, \\
\hline ** Utilisation comme médicament anticancéreux ou non & & Humira, Xarelto, Lucentis & \\
\hline
\end{tabular}


Tableau 6

TOP 20 des coûts des médicaments selon l'art. 71 et dans l'application régulière, Helsana 2012.

\begin{tabular}{|c|c|c|c|c|}
\hline Rang & Médicament & $\begin{array}{l}\text { Coûts selon } \\
\text { l'art. } 71, \text { CHF }\end{array}$ & $\begin{array}{l}\text { Coûts dans l'application } \\
\text { régulière, CHF }\end{array}$ & $\begin{array}{l}\text { Rapport entre les coûts: } \\
\text { art. } 71 \text { et régulière }\end{array}$ \\
\hline 1 & Avastin* & 1586900 & 5209400 & $1: 3,3$ \\
\hline 2 & Mabthera** & 1434200 & 8388900 & $1: 5,8$ \\
\hline 3 & Revlimid* & 690700 & 7797800 & $1: 11$ \\
\hline 4 & Jevtana* & 641000 & 661000 & $1: 1$ \\
\hline 5 & Zytiga* & 461500 & 2889700 & $1: 6,2$ \\
\hline 6 & Privigen & 267600 & 3814300 & $1: 14$ \\
\hline 7 & Velcade* & 240800 & 3841100 & $1: 16$ \\
\hline 8 & Yondelis* & 173500 & 230500 & $1: 1,3$ \\
\hline 9 & Ribomustin* & 137800 & 505800 & $1: 3,6$ \\
\hline 10 & Alimta* & 137000 & 3727200 & $1: 27$ \\
\hline 10-12 & Divers & 734000 & 8376500 & $\begin{array}{l}\text { Kiovig, Yervoy*, Erbitux*, Vidaza*, } \\
\text { Scenesse, Botox, Caprelsa*, llomedin, } \\
\text { Pegasys** }\end{array}$ \\
\hline & Total & 6505000 & 41601100 & $1: 6,4$ \\
\hline
\end{tabular}

Tableau 7

Médicaments ciblés Oncologie, Helsana 2012

\begin{tabular}{lllll}
$\begin{array}{l}\text { Médicaments ciblés } \\
\text { Oncologie }\end{array}$ & $\begin{array}{l}\text { Enregistrement } \\
\text { Swissmedic }\end{array}$ & Cité dans la LS & $\begin{array}{l}\text { Indications à charge } \\
\text { des caisses-maladie }\end{array}$ & $\begin{array}{l}\text { Demande d'indication } \\
\text { selon l'art. 71 a/b OAMal }\end{array}$ \\
\hline 39 & 24 & 22 & 45 & 189
\end{tabular}

Les TOP 20 établis par le service statistique de Helsana ne montrent qu'un petit extrait, car des demandes de prise en charge des coûts selon l'art. $71 \mathrm{a} / \mathrm{b}$ OAMal ont été déposées pour un total de 284 médicaments différents. Sur ce total, 39 étaient des médicaments ciblés dans le domaine de l'oncologie. Selon la liste des spécialités (LS), ces 39 médicaments ciblés sont obligatoirement à charge des caisses-maladie dans 45 indications. Or le corps médical a formulé des demandes pour 189 indications (tableau 7).

Le tableau 7 montre clairement que les médicaments ciblés qui visent des récepteurs bien précis et non des maladies peuvent notamment être utilisés dans de très nombreuses indications. Cela crée un grand nombre d'entités secondaires comme options thérapeutiques qui sont essentiellement utilisées dans le domaine hors étiquette (Off-label use). Il manque aujourd'hui un concept des autorités quant à la façon d'orienter vers le processus d'autorisation régulier ce type de médicaments, où ce n'est plus la maladie, mais les principes actifs qui sont prioritaires.

\section{Références}

1 www.vertrauensaerzte.ch $\rightarrow$ Compétence professionnelle $\rightarrow$ Evaluation du bénéfice selon art. $71 \mathrm{a} / \mathrm{b}$ OAMal

2 Seiler B, Fries R, Honegger H. Evaluation du bénéfice des médicaments hors étiquette. Bull Méd Suisses. 2012;93(19):723-5.

Seiler B et al. Therapeutischer Nutzen eines Medikamentes. SSSP 2011; 112.

3 ASSM .Potentiel et limites de la «médecine individualisée» (personalized medicine). Bull Méd Suisses. 2012; 93(50):1856, chap. 4.7.4.

4 Striking a fair deal in orphan drugs. European Biotechnology. 2013; Vol. 12. No. 6-7.

5 Tribunal des assurances du canton. AG, $3^{\text {ème }}$ Cour, jugement du 2.4.2013.

\section{Articles interactifs}

Vous souhaitez commenter cet article? II vous suffit pour cela d'utiliser la fonction «Ajouter un commentaire» dans la version en ligne. Vous pouvez également consulter les remarques de vos confrères sous: www.bullmed.ch/ numero-actuel/articles-interactifs/ 